\title{
Contralateral Pneumothorax Mimicking Bilateral Pneumothoraces in a Patient with Autopneumonectomy
}

\author{
Ye Kyeong Jun, ${ }^{1}$ Jeong Min Ko, ${ }^{1}$ Hyun Jin Park, ${ }^{1}$ Jun Hyun Baik, ${ }^{1, *}$ and Deog Gon $\mathrm{Cho}^{2}$ \\ ${ }^{1}$ Department of Radiology, College of Medicine, The Catholic University of Korea, Seoul, Republic of Korea \\ ${ }^{2}$ Department of Thoracic Surgery, College of Medicine, The Catholic University of Korea, Seoul, Republic of Korea \\ "Corresponding author: Jun Hyun Baik, Department of Radiology, College of Medicine, St. Vincent's Hospital, The Catholic University of Korea, Seoul, Republic of Korea. Tel: \\ +82-312497484, Fax: +82-312475713, E-mail: jhbaik@catholic.ac.kr
}

Received 2016 May 04; Revised 2016 June 01; Accepted 2016 June 12.

\begin{abstract}
Contralateral pneumothorax crossing the midline in a patient with autopneumonectomy can mimic bilateral pneumothoraces on chest radiography. In this study, we report a rare case of right pneumothorax mimicking bilateral pneumothoraces in a patient with left autopneumonectomy. A 37-year-old man complained of progressive chest pain and dyspnea. The initial chest radiography revealed visible visceral pleura with air in bilateral upper pleural spaces, suggesting bilateral pneumothoraces. Mediastinal structures were shifted to the left side and increased opacities with internal tubular and cystic radiolucencies were noted in the left lower lung zone. After immediate placement of a drainage tube into the right pleural space, the patient underwent chest computed tomography (CT). CT scan revealed a destroyed and totally collapsed left lung and hyper-inflated right lung with right pneumothorax crossing the midline. The contralateral pneumothorax was then gradually absorbed by keeping the chest tube in the right hemithorax. No pneumothorax recurred in 2 years of follow-up. A greater awareness of this rare condition is important in clinical practice, as catastrophic consequences can occur if a chest tube is blindly placed in the autopneumonectomy site.
\end{abstract}

Keywords: Contralateral Pneumothorax, Autopneumonectomy, Chest Radiograph, Computed Tomography, Case Report

\section{Introduction}

An autopneumonectomy is a condition of unilaterally destroyed lung due to variable causes, such as progressively destructive pulmonary infections, fibroatelectasis, primary lung tumors, and mediastinal masses. Volume loss at the autopneumonectomy site will lead to progressive mediastinal shifting and contralateral lung herniation into the autopneumonectomy space (1). A contralateral pneumothorax crossing the midline in a patient with autopneumonectomy could mimic bilateral pneumothoraces on chest radiography and is extremely rare $(2,3)$. We describe a case of right pneumothorax mimicking bilateral pneumothoraces in a patient with left autopneumonectomy. This case report focuses on chest radiography and computed tomography (CT) features. This study was approved by our institutional review board, and the requirement for informed consent was waived.

\section{Case Presentation}

A 37-year-old man was admitted to the emergency room because of an abrupt onset of right chest pain with accompanying dyspnea. The pain propagated to the left chest wall. His past medical history included Mycobacterium tuberculosis infection and chronic obstructive pulmonary disease. He also suffered from pneumothorax seven years prior to presentation. His vital signs were stable except for a mildly increased respiratory rate of 20 breaths/minute. Laboratory studies including arterial blood gas studies were all within normal range. Considering his past medical history of pneumothorax, chest radiography was performed with the suspicion of pneumothorax.

Chest radiography revealed visible visceral pleura with air in bilateral upper pleural spaces, suggesting bilateral pneumothoraces (Figure 1). Mediastinal structures including the trachea and heart were shifted to the left side and the left diaphragm was elevated. Increased opacities with internal tubular and cystic radiolucencies were noted in the left lower lung zone. After immediate placement of a drainage tube into the right pleural space, the patient underwent chest CT exam. The lung window setting of the chest CT scans revealed a destroyed and totally shrunken left lung with cicatricial bronchiectasis and calcifications and a severely displaced mediastinal structure on the left side. There was also an anteriorly herniated hyper-inflated right lung with a right pneumothorax that crossed the midline (Figure 2). The shadow of the lung parenchyma and the pneumothorax on the left upper lung field that appeared on chest radiography was actually the shadow of the contralateral side of the lung and pneumothorax that crossed the midline. There was no evidence of bulla 


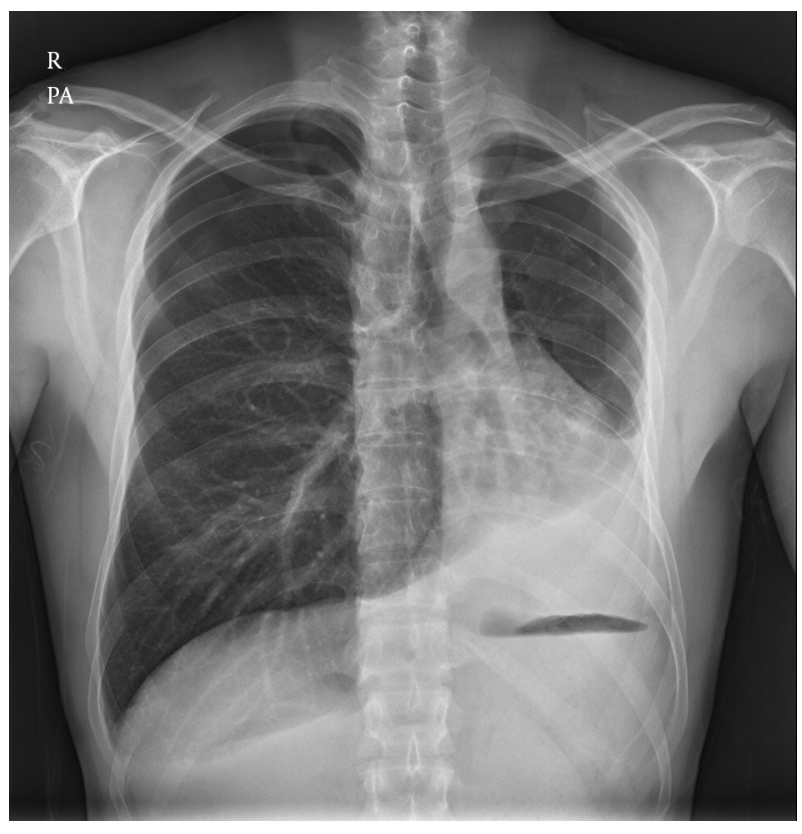

Figure 1. A 37-year-old man who was admitted to the emergency room because of an abrupt onset of right chest pain with accompanying dyspnea. Chest X-ray reveals visible visceral pleura with air in bilateral upper pleural spaces, suggesting bilateral pneumothoraces. Mediastinal structures including the trachea and heart are shifted to the left side and the left diaphragm is elevated. Increased opacities with internal tubular and cystic radiolucencies are noted in the left lower lung zone.

or other abnormal findings in the right lung. The pneumothorax was gradually absorbed, and the chest tube was removed on the sixth hospital day. No pneumothorax recurrence occurred in 2 years of follow-up.

\section{Discussion}

Various progressively destructive pulmonary infections, fibroatelectasis, primary lung tumors and mediastinal masses could be possible causes of a unilaterally destroyed lung, a condition known as autopneumonectomy. The cause of this patient's left lung autopneumonectomy is not clear but considering his past medical history of Mycobacterium tuberculosis infection and his radiological findings, the left lung may have been destroyed by tuberculosis infection. Total destruction of the whole lung is not an uncommon finding in the end stages of tuberculosis (4). Such damage is caused by the combination of parenchymal and airway involvement with pulmonary destruction, bronchiectasis, and fibrosis. In addition to primary or post-primary tuberculosis, secondary pyogenic or fungal infection can also contribute to lung destruction (4).

After surgical pneumonectomy, if the post pneumonectomy space becomes obliterated with continuous
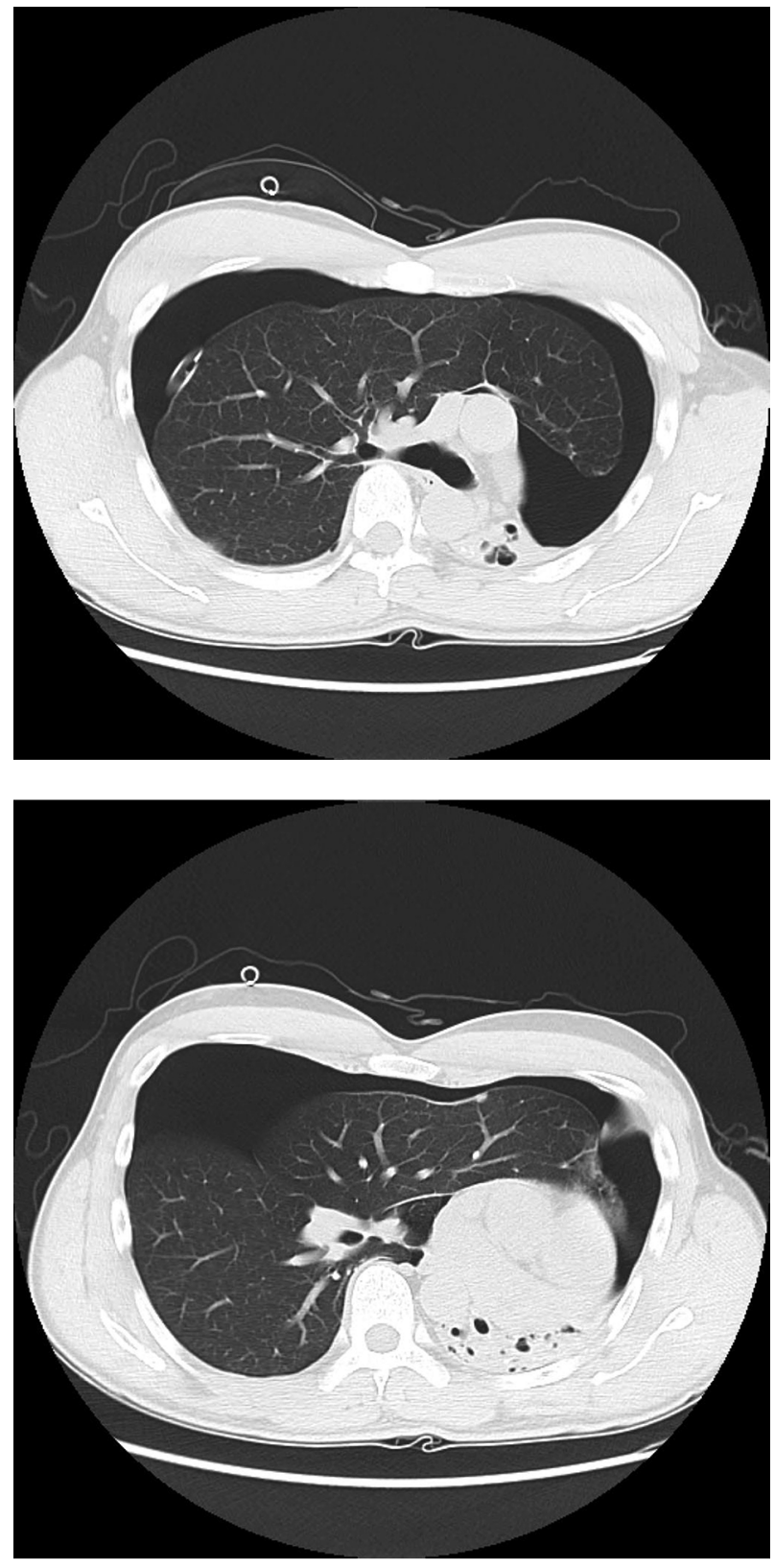

Figure 2. The lung window setting of chest CT scan reveals a destroyed and totally shrunken left lung with cicatricial bronchiectasis and calcifications and a severely displaced mediastinal structure on the left side. There was also a hyper-inflated right lung with a right pneumothorax crossing the midline.

absorption of air and fluid in this space, simultaneously a progressive mediastinal shift and hyperinflation and herniation of the contralateral lung into this space occur(1). In this situation, the heart rotates posteriorly while the contralateral lung herniates across the midline to a position anterior to the heart and aorta (5). Posterior herniation of 
the contralateral lung across the midline posterior to the heart also occurs in patients with a left pneumonectomy $(6,7)$. In the case of autopneumonectomy, a similar process with progressive mediastinal shift and contralateral lung herniation into the autopneumonectomy space may occur.

If contralateral pneumothorax develops in this situation, pleural air can appear in both hemithoraces, and the radiological appearance of the chest can mimic bilateral pneumothoraces. A mediastinal shift, abnormal basithoracic opacities, elevation of the diaphragm, and tracing of bronchovascular markings can be helpful for differentiating contralateral pneumothorax with autopneumonectomy from bilateral pneumothoraces. Visible visceral pleura at the same level of both hemithoraces may also be a helpful finding.

Contralateral pneumothorax is a rare complication after pneumonectomy, with an incidence of $0.3 \%$ to $1.2 \%$ (810) and an overall mortality of approximately 50\% (9). A number of cases of contralateral pneumothorax after pneumonectomy have been reported (10-13). However, there are few reports of contralateral pneumothorax in a condition of autopneumonectomy. To the best of our knowledge, only two cases have been reported. Kato et al. reported a case of unilateral spontaneous pneumothorax mimicking bilateral pneumothoraces in a condition of autopneumonectomy, but initial chest radiography and CT images were not included in the study (2). They initially misdiagnosed the patient with bilateral pneumothoraces and inserted chest tubes on both sides. Belatedly, fiberoptic bronchoscopy revealed interruption of the left main stem bronchus and a displaced right middle lobe into the left hemithorax. Recently, a case of spontaneous pneumothorax in a patient with unilateral agenesis of the lung was reported (3). The chest radiography showed bilateral pneumothoraces, a left basithoracic opacity and an abnormal cardiac silhouette. The chest CT scan revealed absence of the left main bronchus and the left lung parenchyma with the existence of a hyper-inflated right lung and a right pneumothorax crossing the midline.

CT imaging is the modality of choice for confirming a contralateral pneumothorax that crosses the midline as it can visualize a hyper-inflated contralateral lung. CT imaging also plays an important role in evaluating causes of autopneumonectomy and delineating the abnormal location of the mediastinum, liver, and spleen that would otherwise not be detected on plain chest radiographs. Before chest tube insertion, a correct diagnosis of contralateral pneumothorax and the location of the mediastinal structures should be confirmed. This is important because catastrophic consequences could occur if the chest tube is blindly placed in the autopneumonectomy site. A previous case study reported that blind placement of the chest tube into the post-pneumonectomy space resulted in perforation of the right ventricle (14).

In conclusion, contralateral pneumothorax crossing the midline in a case of autopneumonectomy could mimic bilateral pneumothoraces on chest radiography. Awareness of this rare condition is important in clinical practice, as catastrophic consequences can result from blindly placing a chest tube into the autopneumonectomy site.

\section{Footnotes}

Authors' Contributions: Study concept: Jun Hyun Baik and Hyun Jin Park; acquisition of data: Ye Kyeong Jun and Jeong Min Ko; analysis and interpretation of data: all authors; drafting of the manuscript: Ye Kyeong Jun; critical revision of the manuscript for important intellectual content: Jeong Min Ko and Hyun Jin Park; administrative, technical, and material; support: Deog Gon Cho; study supervision: Jun Hyun Baik

Financial Disclosure: None declared. Funding/Support: None declared.

\section{References}

1. Chae EJ, Seo JB, Kim SY, Do KH, Heo JN, Lee JS, et al. Radiographic and CT findings of thoracic complications after pneumonectomy. $R a$ diographics. 2006;26(5):1449-68. doi: 10.1148/rg.265055156. [PubMed: 16973775].

2. Kato R, Kobayashi T, Kawamura M, Kikuchi K, Kobayashi K. Unilateral spontaneous pneumothorax mimicking bilateral pneumothorax. CHEST Journal. 1993;104(5):648b.

3. Hentati A, Neifar C, Abid W, M'Saad S. Left lung agenesis discovered by a spontaneous pneumothorax in a 20-year-old girl. Lung India. 2016;33(2):205-7. doi: 10.4103/0970-2113.177443. [PubMed: 27051112].

4. Kim HY, Song KS, Goo JM, Lee JS, Lee KS, Lim TH. Thoracic sequelae and complications of tuberculosis. Radiographics. 2001;21(4):839-58. doi: 10.1148/radiographics.21.4.g01jl06839. [PubMed: 11452057].

5. Goodman LR. Postoperative chest radiograph: II. Alterations after major intrathoracic surgery. AJR Am J Roentgenol. 1980;134(4):803-13. doi: 10.2214/ajr.134.4.803. [PubMed: 6767369].

6. Biondetti PR, Fiore D, Sartori F, Colognato A, Ravasini R, Romani S. Evaluation of post-pneumonectomy space by computed tomography. J Comput Assist Tomogr. 1982;6(2):238-42. [PubMed: 7076915].

7. Maniwa T, Saito Y, Saito T, Kaneda H, Imamura H. Evaluation of chest computed tomography in patients after pneumonectomy to predict contralateral pneumothorax. Gen Thorac Cardiovasc Surg. 2009;57(1):28-32. doi: 10.1007/s11748-008-0322-z. [PubMed: 19160008].

8. Algar FJ, Alvarez A, Salvatierra A, Baamonde C, Aranda JL, LopezPujol FJ. Predicting pulmonary complications after pneumonectomy for lung cancer. Eur J Cardiothorac Surg. 2003;23(2):201-8. [PubMed: 12559343].

9. Blalock JB. Contralateral pneumothorax after pneumonectomy for carcinoma. Dis Chest. 1960;37:371-7. [PubMed: 13801275].

10. Hubbard JG, Nkere UU, Bhatnagar NK. Spontaneous contralateral pneumothorax following pneumonectomy. Postgrad Med J. 1997;73(856):107-8. [PubMed: 9122088]. 
11. Deutsch MA, Martetschlaeger F, Muenzel D, D’Haese JG, Krane M, Bauernschmitt R, et al. Combined spontaneous contralateral pneumothorax and post-pneumonectomy mediastinal shift-associated dextrocardia. Thorac Cardiovasc Surg. 2011;59(1):60-2. doi: 10.1055/s0030-1250202. [PubMed: 21243579].

12. Furukawa M, Oto T, Toyooka S, Soh J, Yamane M, Miyoshi S. Contralateral pneumothorax in bullous lung after pneumonectomy: report of two cases. Gen Thorac Cardiovasc Surg. 2013;61(1):35-7. doi: 10.1007/s11748-012-0112-5. [PubMed: 22614524].

13. Matsuoka K, Ito A, Murata Y, Kuwata T, Takasaki C, Imanishi N, et al. Four cases of contralateral pneumothorax after pneumonectomy. Ann Thorac Surg. 2014;98(4):1461-3. doi: 10.1016/j.athoracsur.2013.12.066. [PubMed: 25282215].

14. Kopec SE, Conlan AA, Irwin RS. Perforation of the right ventricle: a complication of blind placement of a chest tube into the postpneumonectomy space. Chest. 1998;114(4):1213-5. [PubMed: 9792599]. 